\section{P193 COMPLICATIONS AFTER THORACOCENTESIS AND SMALL BORE INTERCOSTAL DRAIN INSERTION: A SINGLE CENTER STUDY FROM THE NORTH EAST OF ENGLAND}

K Jackson, A Aujayeb. Northumbria Healthcare NHS Trust, Newcastle upon Tyne, UK

\subsection{6/thorax-2021-BTSabstracts.302}

Introduction There are no prospective studies looking at complications of pleural procedures. Previous British Thoracic Society Pleural audits and retrospective case series inform current practice. Incidence of any complication is between 1$15 \%$. We sought to add to the existing literature and inform local practice with regards to intercostal drains and thoracocenteses.

Methods Local Caldicott approval was sought for a review of all inpatient adult pleural procedures coded as 'T122 drainage of pleural cavity' and 'T124 insertion of tube drain into pleural cavity'. Those undergoing thoracocentesis (all with a Rocket $6 \mathrm{Fg}$ catheter) and intercostal drain insertion (ICD, all with Rocket $12 \mathrm{Fg}$ drain) were identified. Continuous variables are presented as mean ( \pm range) and categorical variables as percentages where appropriate.

Results 1159 procedures were identified. 199 and 960 were done for pneumothorax and effusions respectively. Mean age was 68.1 years (17-97). There were 280 thoracocenteses and 879 ICDs. Bleeding occurred in $6(0.5 \%)$, all ICDs (clotting and platelets were within normal range, 1 patient was on aspirin, 1 on aspirin and clopidogrel). All settled except for one who had intercostal artery rupture needing cardiothoracic intervention (no anti-coagulation). 9 pneumothoraces occurred $(0.78 \%)$ in 7 ICDs and 2 aspirations). There were 3 definite pleural space infections $(0.3 \%)$ with 3 ICDs. Fall out rates for ICDs were 35 (3\%). 9 were not sutured, out of those 7 inserted in the Accident and Emergency department, out of hours. All others 'came out' due to patient factors. (previous quoted rates up to 14\%). Surgical emphysema occurred in 43 (41 ICDs), 3.7\%. 8 were due to fall outs and 3 required surgical intervention. There were no re-expansion pulmonary edema and no direct deaths.

Conclusions Complication rates of ICD and thoracocenteses are low. Checklists might help to remind operators of the need for suturing. Limitations of this study are its retrospective nature, and reliance on correct hospital coding.

\section{P194 SEPTATION FORMATION FOLLOWING PLEURAL INTERVENTION}

H Rai, N Sarnrak, E Graham, A Ghoshal, T Nicholson, LM Taylor, JP Corcoran, C Daneshvar. University Hospitals Plymouth NHS Trust, Plymouth, UK

\subsection{6/thorax-2021-BTSabstracts.303}

Background Recurrent pleural intervention may complicate the pleural space by inducing pleural inflammation with subsequent septation formation. We evaluated our five year experience in the incidence of pleural septations in patients undergoing pleural interventions.

Method We retrospectively identified patients who underwent thoracic ultrasound (TUS) in our pleural service from our reporting database between August 2015 and February 2021. Categorical reporting of the presence of septations was used, reporting septations as either present or absent. Repeated
TUS, types of pleural interventions, and time between these interventions were analysed.

Results Of the 2737 index TUS performed, we recorded whether septations were present or not in 2684 (98\%) patients. Of these, septations were present in $715 / 2684$ (26.6\%: 95\% CI 25-28.3\%) cases.

In 297 patients with $>1$ TUS reports, 187 underwent an intervention at the index visit. At baseline, septations were present in 39/187 (20.9\%) of these patients. Of the remaining $148 / 187(79.1 \%)$ patients without septations on index scan, 24/148 (16.2\%; 95\% CI 10.7-23.2) reported the formation of new septations at the second TUS visit at a median [interquartile range] time interval of 21 [9-63] days.

No association was seen between the type of intervention and development of septations [chest drain 14.2\% (1/7), diagnostic aspiration only $22.2 \%(6 / 27)$, therapeutic aspiration $15.3 \%$ ( 17/111) $\mathrm{p}=0.68$ ].

No difference was observed in those patients with serial scans, not undergoing intervention, with new septations reported in $15 / 75(20 \%)(p=0.48)$, while a shorter time interval between scans reporting conversion to septations (median [IQR] 7 [2.25-57] days $(\mathrm{p}=0.04))$ was noted.

Conclusion Overall, in this large cohort of patients seen through our pleural service, septations were present in a quarter of baseline thoracic ultrasound examinations. Septations formed with or without intervention in around 1 in 5 patients. Understanding this further has significant implications for diagnostic and management pathways.

\section{P195 PLEURAL EFFUSIONS ASSOCIATED WITH PERICARDITIS OR MYOCARDITIS}

R Ahmed, R Scott, K Jackson, A Aujayeb. Northumbria HealtCare NHS Foundation Trust, Newcastle, UK

\subsection{6/thorax-2021-BTSabstracts.304}

Introduction A literature search over the last decade found 1 paper regarding pleural effusions in pericarditis/myocarditis. 94 pleural effusions were described in 177 patients (53\%). They were predominantly bilateral, associated with $\mathrm{C}$ reactive protein (CRP) levels and female sex. ${ }^{1}$ No local review has ever been performed. We sought to add to the literature and inform local practice.

Methods With Caldicott approval, a review of all cardiac magnetic resonance imaging (cMR) records from the local cardiological database was performed. Pericarditis/myocarditis cases were analysed further to determine presence of pleural effusions. Those were analysed for basic demographics, pleural interventions and outcomes. Continuous variables are presented as mean ( \pm range) and categorical variables as percentages where appropriate.

Results 4368 cMRs were reviewed (July 2016-July 2020); 82 (1.9\%) patients had pericarditis/myocarditis; 28 (33\%) had pleural effusions on contemporaneous imaging. Mean age was 63.1 years (range 24-83); 15 were female, and 19 male. Diagnoses were viral (1), rheumatological (2), amyloidosis (2), listeria (1) and the rest idiopathic (22). 3 effusions were only left sided, 1 right sided and 24 bilateral. 7 pleural taps were performed, 1 for a unilateral effusion and 6 for one side being bigger than the other. The mean $\mathrm{Ph} 7.46$ (7.33-7.6), mean LDH 210 (74-393 U/L), mean fluid protein 36.1 (19-56 g/L) [4 effusions exudative/3 transudative], mean glucose 5.8 (4.8$6.8 \mathrm{mmol} / \mathrm{L})$, all cytologies were negative. 6 patients 